\title{
Dialkylzincs in Radical Reactions
}

\author{
Samantha Bazin, Laurence Feray, and Michèle P. Bertrand*
}

\begin{abstract}
This review describes recent developments in the use of dialkylzincs in radical reactions. In most of the processes reported therein, dialkylzincs act simultaneously as sources of alkyl radicals, as Lewis acids, and as chain transfer agents. A parallel is drawn with the behaviour of trialkylboranes in similar reactions.
\end{abstract}

Keywords: Conjugate addition · Dialkylzinc · Imines · Radical addition · Radical initiator ·

Radical-polar crossover reactions

\section{Introduction}

Dialkylzincs are moisture- and air-sensitive organometallic species which are generally used in carefully deoxygenated medium. Their fast reaction with oxygen has been known since their discovery [1]; however, the mechanism of their oxidation was only elucidated a hundred years later, in 1968, thanks to the work of Davies and Roberts on bimolecular homolytic substitution at metal centres [2]. With the exception of a few attempts to initiate free-radical polymerizations [3], the use of dialkylzincs as a source of alkyl radicals in the presence of oxygen stimulated the interest of radical chemists only much later. It is known that the zinc/ iodine exchange can be promoted by $\mathrm{Cu}(\mathrm{I})$, $\mathrm{Ni}(0)$ or $\mathrm{Pd}(0)$, and a radical mechanism has been proposed for these reactions [4]. It has also been observed that air accelerates the formation of zinc carbenoids from diethylzinc and dihalomethanes [5]. This review intends to emphasize the benefits of using these organometallic species as mediators in radical additions. The first advantage resides in the proposal of environmentally friendly tin-free procedures. The second one is a consequence of their aptitude to act as chain transfer agents through their fast reaction with heteroatom-centred radical species, which enables the performance of radical-polar crossover reactions.

\section{Initiation of the Reduction of Alkyl Halides with Tributyltin Hydride}

Diethylzinc was used by Ryu et al. to initiate tin hydride-mediated reduction of alkyl halides [6]. As exemplified in Scheme 1 with the reduction of adamantylbromide, the reaction proceeds in non-degassed solvent in the presence of $5 \mathrm{~mol} \%$ of $\mathrm{Et}_{2} \mathrm{Zn}$. The yield in adamantane is quantitative when air is added to the reaction medium.

\section{Radical Additions to $\mathbf{C}=\mathbf{N}$ Bonds}

The reactivity of dialkylzincs with respect to imino group was first investigated by Van Koten and coworkers [7]. Dialkylzincs react with 1,4-diaza-1,3-butenes under inert atmosphere to give either a Calkylated or a N-alkylated product depending on the nature of the alkyl group in the organometallic species (Scheme 2).
Mechanistic studies by EPR spectroscopy have led to the proposal of two possible mechanisms. The first one would proceed through the cage recombination of the persistent radical species $\mathbf{2}$ with the alkyl radical (path (a)). The second one would involve an electron transfer from $\mathbf{2}$ to $\mathbf{1}$, prior to the recombination of a carbanion with the cationic complex $\mathbf{5}$ (path (b)). Once freed from the solvent cage, $\mathbf{2}$ is in equilibrium with its dimer $\mathbf{3}$.

The behaviour of glyoxylate imines with respect to diethylzinc depends strongly on the experimental conditions. Under inert atmosphere, they lead to azetidinones through a reaction involving the transfer of the ethyl group to nitrogen (the mechanism is likely to involve polar species, but it might also involve a cage radical recombination). The resulting zinc enolate adds to the complexed imine (6) to form the $\beta$ lactam (Scheme 3) [8].

In contrast, when air is added to the reaction medium, the $\mathrm{N}$-alkylated product $\mathbf{8}$ is formed in very low amounts, the major product being the C-alkylated product 7 (Scheme 4). When diethylzinc is used alone, the amount of $\mathbf{8}$ depends on the amount of oxygen in the reaction medium. Moreover when six equivalents of $t$-butyl iodide are added to the reaction medium, $\mathbf{8}$
${ }^{*}$ Correspondence: Prof. Dr. M.P. Bertrand Laboratoire de Chimie Moléculaire Organique UMR 6517, Boite 562

Université Paul Cézanne, Aix-Marseille III Faculté des Sciences St Jérôme Avenue Escadrille Normandie-Niemen 13397, Marseille, Cedex 20, France Tel.: + 33491288597

E-Mail: michele.bertrand@univ.u-3mrs.fr

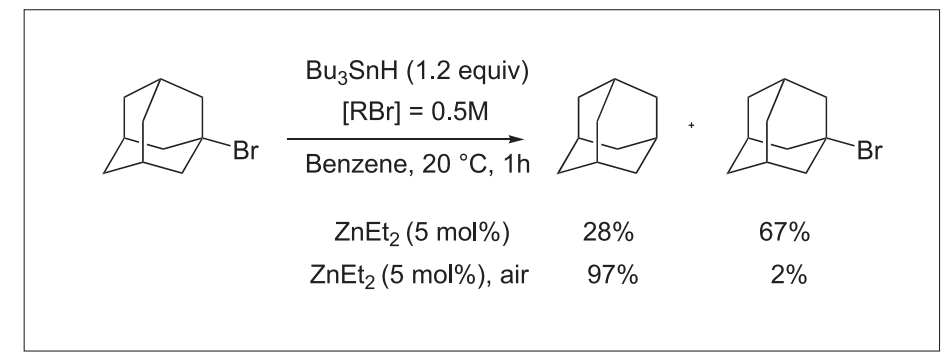

Scheme 1. 


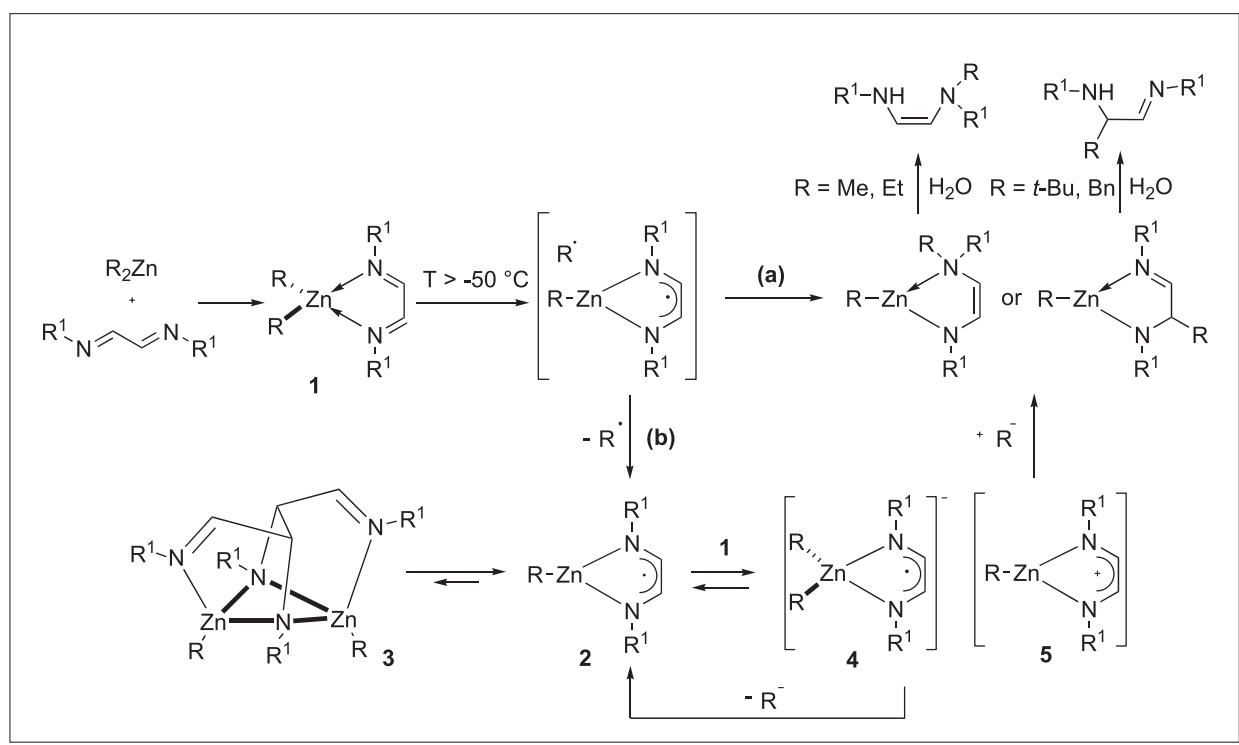

Scheme 2.

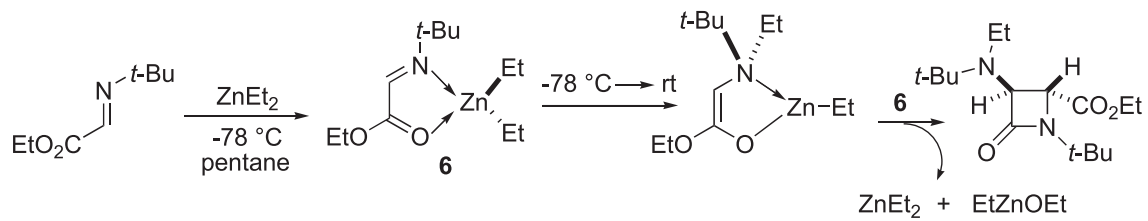

Scheme 3.

$$
\begin{aligned}
& t-\mathrm{Bul}\left(0 \text { equiv), } 20{ }^{\circ} \mathrm{C}\right. \\
& t \text { - } \mathrm{Bul}\left(6 \text { equiv), }-40{ }^{\circ} \mathrm{C}\right.
\end{aligned}
$$

Scheme 4.

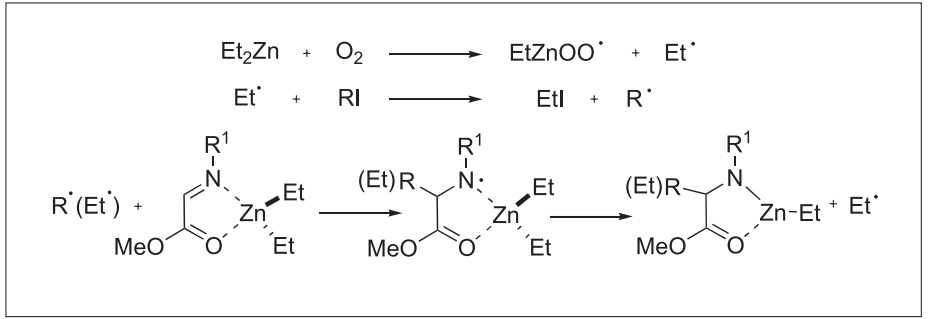

Scheme 5.

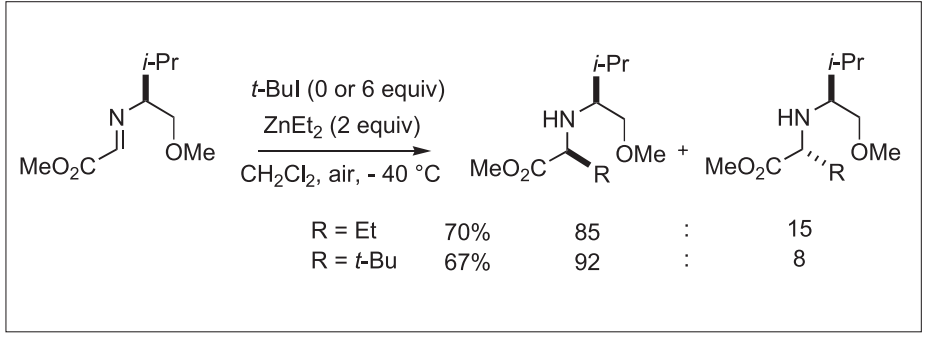

Scheme 6 . is no more detected, the only product is the adduct of $t$-butyl radical to the $\mathrm{C}=\mathrm{N}$ bond [9]. This argues against 8 originating from a cage radical mechanism.

Since a $\mathrm{Zn}$ /iodine exchange is highly unlikely in the case of a tertiary alkyl iodide, Bertrand and coworkers have proposed the mechanism shown in Scheme 5, in which $\mathrm{Et}_{2} \mathrm{Zn}$ plays simultaneously the role of initiator by reacting with oxygen, the role of Lewis acid by activating the reactivity of the $\mathrm{C}=\mathrm{N}$ bond with respect to the nucleophilic alkyl radical, and finally the role of chain transfer agent, by reacting with the incipient aminyl radical according to an $\mathrm{S}_{\mathrm{H}} 2$ process. Ethyl radical, vector of the chain reaction, reacts via iodine atom transfer from the alkyl iodide. This mechanism is very close to the mechanism generally accepted for the triethylboranemediated radical addition to imines [9bd][10].

A higher level of stereoinduction is recorded with the imines derived from valinol owing to the chelating properties of the auxiliary (Scheme 6).

The methodology has been successfully applied to other imino group containing radical acceptors such as oxime ethers and hydrazones [9b,d]. These compounds are more reactive than the corresponding glyoxylic imines. This raises the problem of chemoselectivity since it is more difficult to avoid the competitive addition of ethyl radical, except by using a large excess of secondary or tertiary alkyl iodides.

Temporary connection to camphorsultam leads to high stereoselectivity (Scheme 7) [11]. The procedure has also been extended to oxime ethers supported on Wang resins [11c].

The reactivity of imino groups is strongly dependent on the nature of the substituents at both nitrogen and carbon. As long as the imino group is made electrophilic by an electron-withdrawing group at carbon, the reactivity of oxime ethers and diphenylhydrazones is very high [9d]. In contrast, dialkylhydrazones such as $\mathbf{9}$ are much less reactive and, owing to the fast consumption of diethylzinc with oxygen, a portionwise addition of an excess of reagents is necessary to achieve the addition in acceptable yields. Similarly, in the absence of activation at carbon, oxime ether $\mathbf{1 0}$ reacts very slowly (Scheme 8) [12].

The use of dimethylzinc as the mediator was investigated by Tomioka and coworkers [13]. In the presence of oxygen, dimethylzinc behaves as a source of methyl radical. Owing to enthalpic factors, methyl radical is far more efficient than ethyl radical to abstract hydrogen atoms from different substrates like ethers [13a,b] and cycloalkanes [13c] (the $\mathrm{C}-\mathrm{H}$ BDE in methane is $440 \mathrm{~kJ} \mathrm{~mol}^{-1}$, it is only $411 \mathrm{~kJ} \mathrm{~mol}^{-1}$ in ethane). As exemplified in Scheme 9, when 


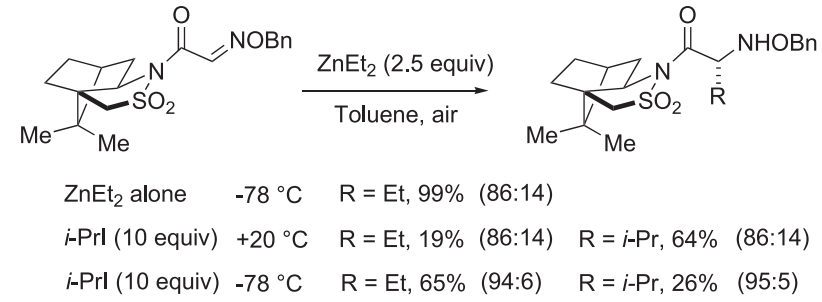

Scheme 7

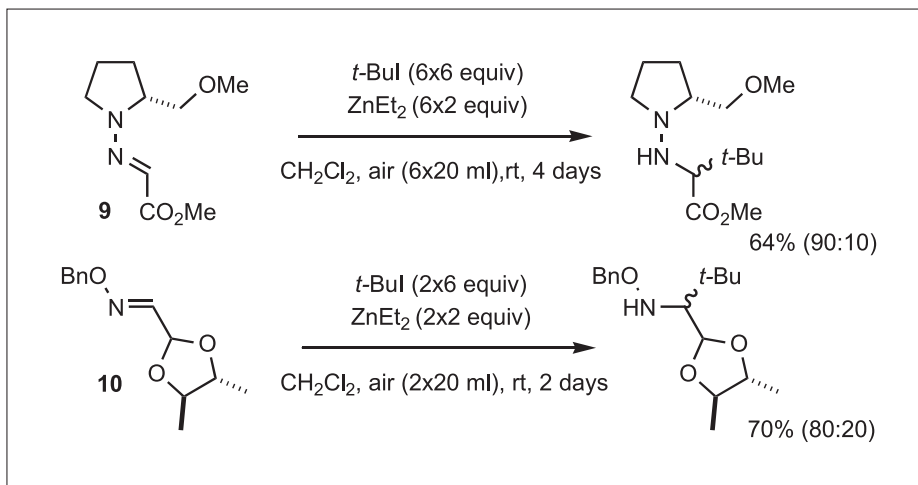

Scheme 8.

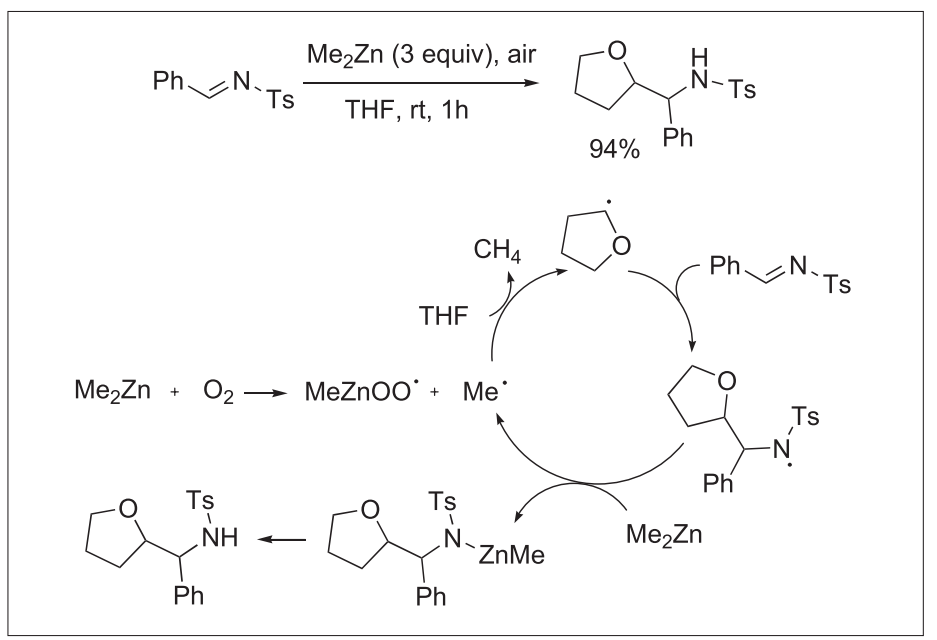

Scheme 9 .

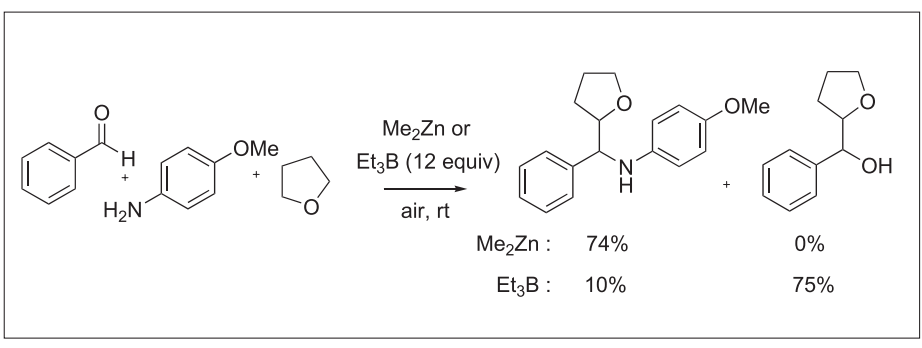

Scheme 10.

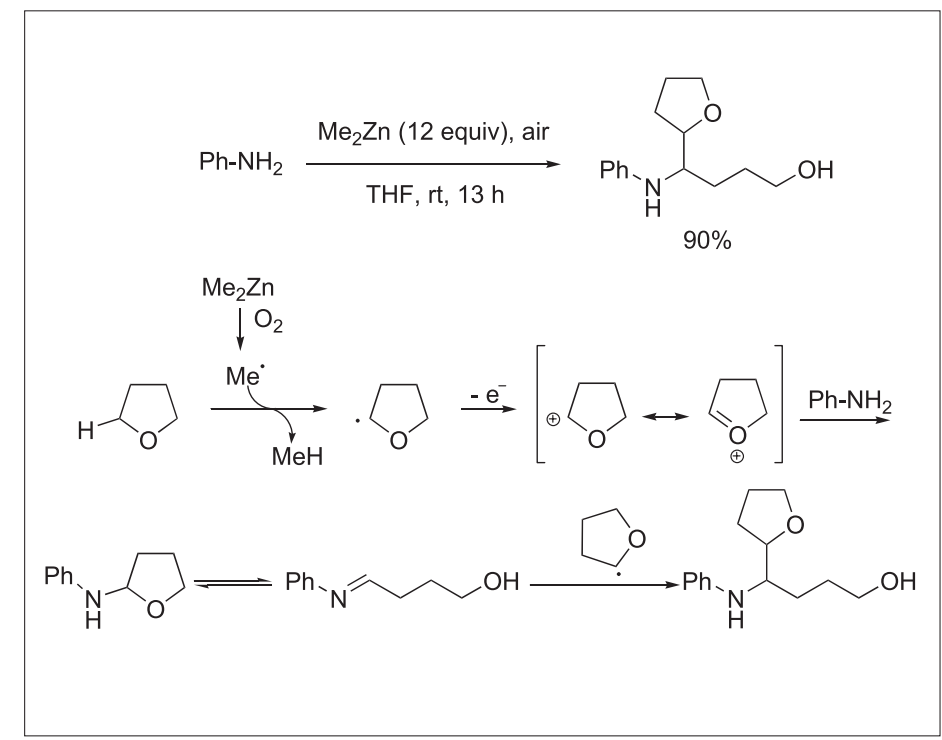

Scheme 11.

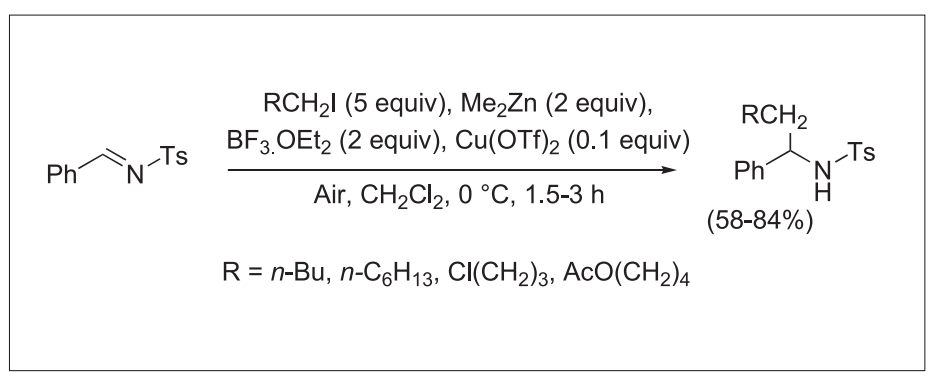

Scheme 12.
THF is used as the solvent nearly quantitative yields in 2-tetrahydrofuranyl radical adduct are isolated. The reaction is accelerated by bubbling air through the reaction medium. No competitive addition of methyl radical is detected, only the most nucleophilic radical in the medium adds to the $\mathrm{C}=\mathrm{N}$ bond. Comparatively, diethylzinc or diisopropylzinc lead to low yields and to competitive addition of ethyl and isopropyl radical, respectively.

In the presence of benzaldehyde and an aromatic amine in solution in THF, dimethylzinc enables the multicomponent reaction to proceed through addition of the $\alpha$-THF radical to the in situ formed imine in good yield, without any competitive addition of the $\alpha$-THF radical to the $\mathrm{C}=\mathrm{O}$ bond (Scheme 10) [13d] In contrast, when triethylborane is used as the mediator, the major product results from addition to the carbonyl group. This multicomponent reaction can be applied to alkoxyamines, hydrazines, and cyclic ethers other than THF. However, hydrazines give moderate yields.

In fact, the dimethylzinc-mediated addition of THF to benzaldehyde proceeds very slowly [14a]. When dimethylzincmediated oxidation of the $\alpha$-THF radical is conducted in the presence of aniline, it leads to the adduct of the $\alpha$-alkoxyalkyl radical to an intermediate iminoalcohol according to Scheme 11 [14b].
The use of dimethylzinc as the mediator instead of diethylzinc enables the addition of alkyl iodides to proceed even with primary alkyl iodides. As stated previously, owing to more favourable enthalpic factors iodine transfer to methyl radical is much faster than iodine transfer to the ethyl radical [15]. The reaction does not proceed in the absence of air, which argues in favour of a radical process. However, the addition of alkyl radicals of low nucleophilicity needs to be accelerated by the addition of Lewis acids. In the presence of $\mathrm{BF}_{3} \cdot \mathrm{OEt}_{2}$ and $\mathrm{Cu}(\mathrm{OTf})_{2}$, the reaction is totally chemoselective with only five equivalents of primary alkyl iodide (Scheme 12). 


\section{Conjugate Radical Additions and Radical-Polar Crossover Reactions}

In the presence of air, diethylzinc mediates radical addition to enones. No additional catalyst is needed [9b]. When diethylzinc is used alone the product resulting from the addition of the ethyl radical to cyclohexanone is isolated in $64 \%$ yield. In the presence of six equivalents of a secondary or a tertiary alkyl iodide, iodine transfer is faster than the addition of ethyl radical and addition products of secondary and tertiary alkyl radicals are observed (Scheme 13). The reaction has been applied to 2-H-pyran-3-ones by Feringa and coworkers [16].

Most likely, the mechanism is similar to that proposed by Brown and Midland for the conjugate addition of boranes [17]. The intermediate enoxyl radical reacts (like previously the aminyl radical) by bimolecular homolytic substitution at zinc to give a polar intermediate; in this case, a zinc enolate, which can be trapped by electrophiles (Scheme 14).
Since homolytic substitution at zinc is easier than homolytic substitution at boron, other acceptors behave like enones with respect to dialkylzinc that do not react in the same way with triethylborane. N-Enoyloxazolidinones are among these. The aptitude of the enoxyl radical to react by homolytic substitution at zinc or boron is commonly correlated to spin delocalization at oxygen. The $\alpha$-imidoyl radicals have a spin density at oxygen intermediate between enoxyl and $\alpha$-alkoxycarbonyl radicals [18]. The behaviour of the latter depends on their substitution, the limits appeared to be very small since secondary $\alpha$-alkoxycarbonyl radicals give rise to homolytic transfer at zinc followed by aldol condensation, whereas tertiary ones do not [19].

The above behaviour of methacrylates is in good agreement with the cascade reaction performed by Chemla and coworkers on ester 11 [20]. Dialkylzinc 14 does not result from the cyclization of a zinc enolate that would lead to the opposite diastereomer, as demonstrated by forming unambiguously the enolate in two steps (carbozincation with tri-n-butylzincate followed by reaction with n-butylzinc bromide). It results from the cyclization of radical $\mathbf{1 2}$, which indirectly confirms that the tertiary radical does not undergoes homolytic substitution at zinc. The reduction of radical $\mathbf{1 3}$ by $\mathrm{Bu}_{2} \mathrm{Zn}$ leads to 14 . The exchange between the two alkyl groups is favoured since $\mathbf{1 4}$ is stabilized by chelation with the carboxylate [21]. Organozinc derivative $\mathbf{1 4}$ can be trapped by different electrophiles to give $\mathbf{1 5}$ in good yields (Scheme 15).

In the 'one-pot' reaction reported by the Takemoto group, the delocalized tertiary $\alpha$-alkoxycarbonyl radical 19 reacts by $\mathrm{S}_{\mathrm{H}} 2$ with diethylzinc to give zinc enolate 20 which is trapped by the $\pi$-allyl palladium complex 17 (Scheme 16) [22].

These results suggest that the tendency of $\alpha$-alkoxycarbonyl radicals to react by homolytic substitution at zinc is also sensitive to their aptitude to form chelated enolates. In contrast to simple $\alpha$-alkoxycarbonyl radicals [17], malonyl radicals have also been

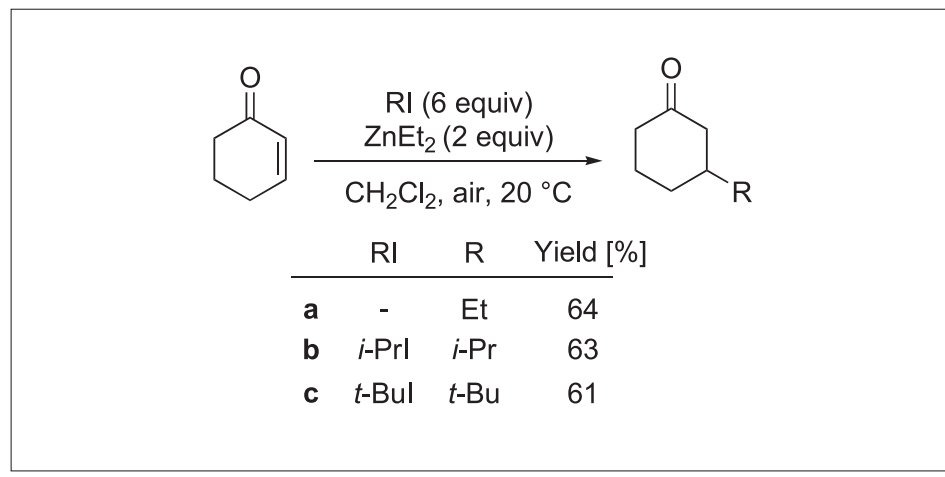

Scheme 13.

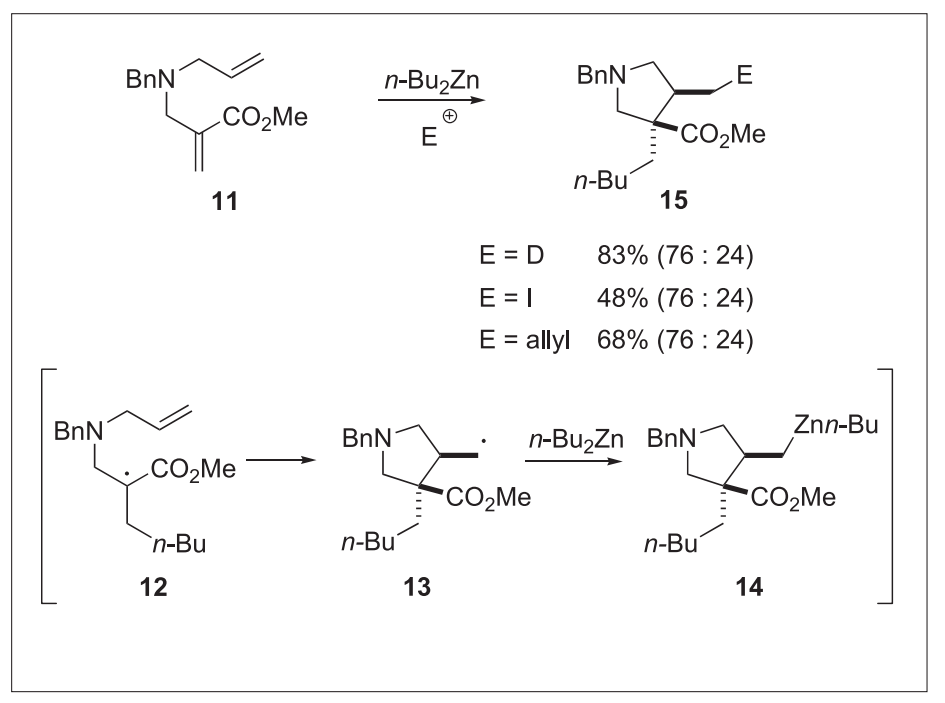

Scheme 15.

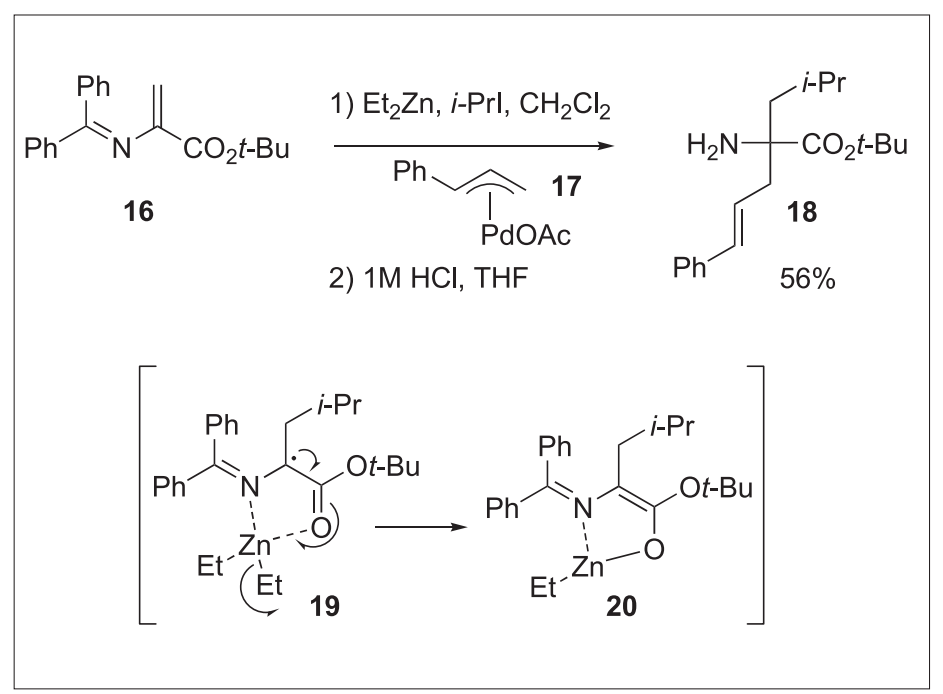

Scheme 16.

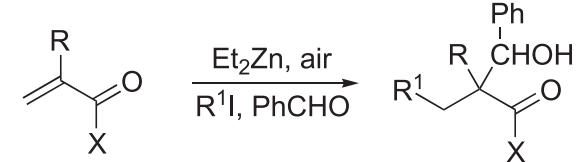<smiles>[R]C=[R]O[Ca]</smiles>

$60-87 \%$

no aldol<smiles>[R]CC([R])=C([X])OCC</smiles> 


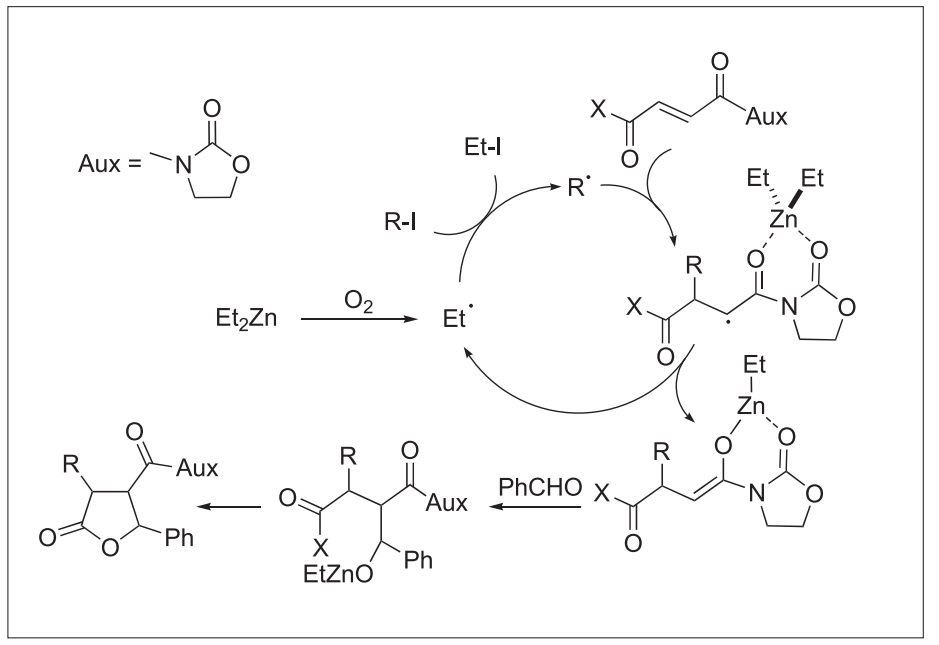

Scheme 17.

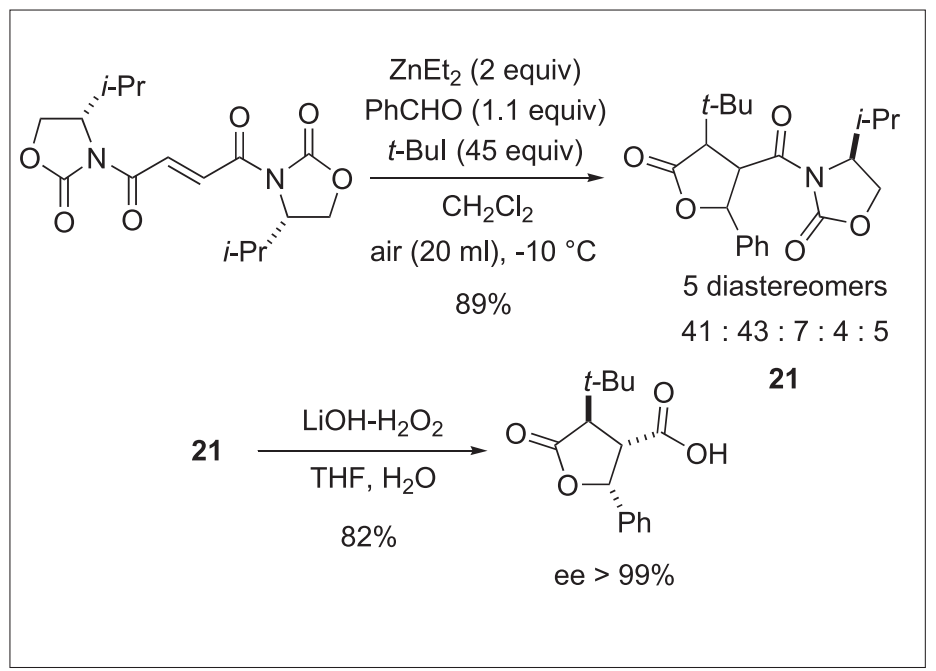

Scheme 18.

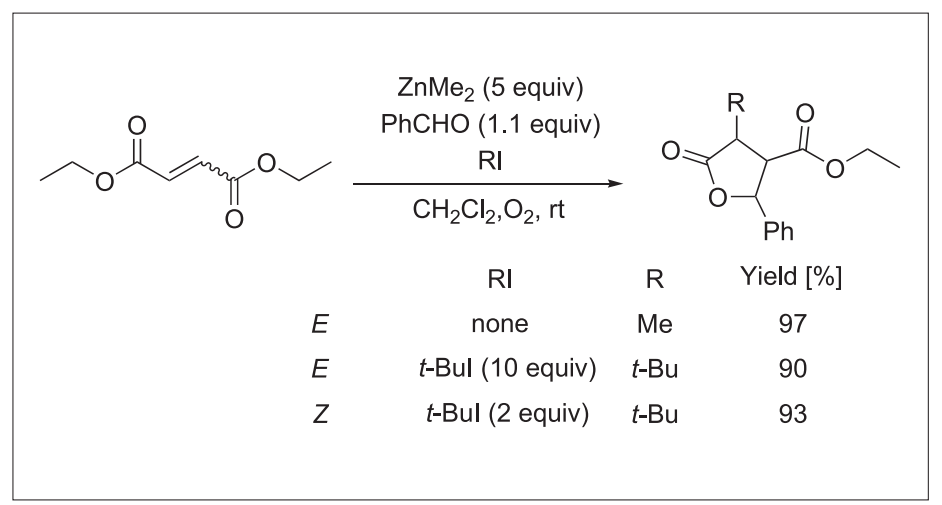

Scheme 19.

reported to form boron enolates via homolytic substitution at triethylborane [23].

Domino reactions leading to $\gamma$-lactones have been performed with fumaric derivatives. As shown in Scheme 17, an additional lactonisation step takes place.

Thanks to epimerization at the chiral centres $\alpha$ to carbonyl groups under the basic conditions used for the cleavage of the auxiliary, optically pure trisubstituted lac- tones have been prepared from optically pure chiral symmetrical bis-N-enoyloxazolidinones (Scheme 18) [24].

The use of dimethylzinc as the mediator enables the formation of methylated lactones from either diethylfumarate or diethyl maleate through the addition of methyl radical. Owing to the acceleration of the iodine transfer reaction with respect to the addition of a methyl radical to the ac- tivated double bond, formation of lactones bearing a $t$-butyl group can be performed in the presence of only two equivalents of $t$-butyl iodide (Scheme 19) [19].

\section{Conclusion}

The need for environmentally friendly radical methodologies has stimulated the search for tin-free procedures. This is probably the main reason why the use of dialkylzincs as mediators is currently being developed. In addition, the aptitude of heteroatom-centred radicals (aminyl and enoxyl radicals) to give rise to homolytic substitution at zinc enables radical-polar crossover reactions to occur through the transformation of a radical intermediate into a polar species (aminozinc, or zinc enolate). Several multi-component domino reactions based on the 'switchable' properties of organozinc compounds have already been proposed.

Received: March 3, 2006

[1] For a history of alkylzincs chemistry, see: D. Seyferth, Organometallics 2001, 20, 2940

[2] a) A.G. Davies, B.P. Roberts, J. Chem. Soc. (B) 1968, 1074; b) A.G. Davies, B.P. Roberts, Acc. Chem. Res. 1972, 387.

[3] J. Lal, J. Polym. Sci., Part A-1 1966, 4, 1163.

[4] For general reviews on the reactivity of dialkyzinc, see: a) P. Knochel, Synlett 1995 , 393; b) P. Knochel, J.J. Almena Perea, P. Jones, Tetrahedron 1998, 54, 8275.

[5] a) S. Miyano, H. Hashimoto, J. Chem. Soc., Chem. Commun. 1971, 1418; b) S. Miyano, H. Hashimoto, Bull. Chem. Soc. Jpn 1973, 46, 892; c) S. Miyano, Y. Izumi, H. Fujii, H. Hashimoto, Synthesis 1977, 700; d) G. Rousseau, N. Slougui, J. Am. Chem. Soc. 1984, 106, 7283.

[6] I. Ryu, F. Araki, S. Minakata, M. Komatsu, Tetrahedron Lett. 1998, 39, 6335.

[7] a) J.M. Klerks, J.T.B.H. Jastrzebski, G. Van Koten, K. Vrieze, J. Organomet. Chem. 1982, 224, 107. b) G. Van Koten, J.T.B.H. Jastrzebski, K. Vrieze, J. Organomet. Chem. 1983, 250, 49; c) M. Kaupp, H. Stoll, H. Preuss, W. Kaim, T. Stahl, G. Van Koten, E. Wissing, W.J.J. Smeets, A.L. Spek, J. Am. Chem. Soc. 1991, 113, 5606; d) E. Wissing, R.W.A. Havenith, J. Boersma, G. Van Koten, Tetrahedron Lett. 1992, 33, 7933; e) E. Wissing, M. Kaupp, J. Boersma, A.L. Spek, G. Van Koten, Organometallics 1994, 13, 2349; f) E. Wissing, S. Van der Linden, E. Rijnberg, J. Boersma, W.J.J. Smeets, A.L. Spek, G. Van Koten, Organometallics 1994, 13, 2602; g) E. Wissing, E. Rijnberg, P.A. Van der Schaaf, K. Van Gorp, J. Boersma, G. Van Koten, Organometallics 1994, 13, 2609. 
[8] a) M.R.P. Van Vliet, J.T.B.H. Jastrzebski, W.J. Klaver, K. Goubitz, G. Van Koten, Recl. Trav. Chim. Pays-Bas 1987, 106, 132; b) For reaction with imino-ketones, see: M.R.P. Van Vliet, G. Van Koten, P. Buysingh, J.T.B.H. Jastrzebski, A.L. Spek, Organometallics 1987, 6, 537.

[9] a) M.P. Bertrand, L. Feray, R. Nouguier, P. Perfetti, Synlett 1999, 7, 1148;. b) M.P. Bertrand, L. Feray, R. Nouguier, P. Perfetti, J. Org. Chem. 1999, 64, 9189; c) M.P. Bertrand, S. Coantic, L. Feray, R. Nouguier, P. Perfetti, Tetrahedron 2000, 56, 3951; d) M.P. Bertrand, L. Feray, S. Gastaldi, C. R. Chimie 2002, 623; e) For closely related studies, see also: N. Singh, R.D. Anand, S. Trehan, Tetrahedron Lett. 2004, 45, 2911.

[10] G.K. Friestad, Tetrahedron 2001, 57, 5461.

[11] a) H. Miyabe, M. Ueda, N. Yoshioka, K. Yamakawa, T. Naito, Tetrahedron 2000, 56, 2413; b) H. Miyabe, C. Ushiro, M. Ueda, K. Yamakawa, T. Naito, J. Org. Chem. 2000, 65, 176; c) H. Miyabe, C. Konishi, T. Naito, Org. Lett. 2000, 2, 1443.

[12] L. Feray, M.P. Bertrand, unpublished results.

[13] a) K.-I.Yamada, H. Fujihara, Y. Yamamoto, Y. Miwa, T. Taga, K. Tomioka, Org. Lett. 2002, 4, 3509; b) K.-I. Yamada, Y. Yamamoto, M. Maekawa, K. Tomioka, $J$. Org. Chem. 2004, 69, 1531; c) K.-I. Yamada, Y. Yamamoto, M. Maekawa, J. Chen, K. Tomioka, Tetrahedron Lett. 2004, 45,
6595; d) K.-I.Yamada, Y. Yamamoto, K. Tomioka, Org. Lett. 2003, 5, 1797.

[14] a) Y. Yamamoto, K.-I. Yamada, K. Tomioka, Tetrahedron Lett. 2004, 45, 795; b) Y. Yamamoto, M. Maekawa, T. Akindele, K.-I. Yamada, K. Tomioka, Tetrahedron 2005, 61, 379.

[15] K.-I. Yamada, Y. Yamamoto, M. Maekawa, T. Akindele, H. Umeki, K. Tomioka, Org. Lett. 2006, 8, 87.

[16] H. Van der Deen, R.M. Kellogg, B.L. Feringa, Org. Lett. 2000, 2, 1593.

[17] a) H.C. Brown, M.M. Midland, Angew. Chem., Int. Ed. Engl. 1972, 11, 692; b) For a review, see: C. Ollivier, P. Renaud, Chem. Rev. 2001, 101, 3415; c) A.-P. Schaffner, P. Renaud, Eur. J. Org. Chem. 2004, 2291.

[18] S. Bazin, L. Feray, J.-V. Naubron, D. Siri, M. Bertrand, Chem. Commun. 2002, 2506.

[19] Samantha Bazin, Thesis Marseille, December 2005.

[20] F. Denes, F. Chemla, J.-F. Normant, Angew. Chem., Int. Ed. 2003, 42, 4043.

[21] For a similar observation, see: A.B. Charette, A. Beauchemin, J.-F. Marcoux, J. Am. Chem. Soc. 1998, 120, 5114.

[22] a) H. Miyabe, R. Asada, K. Yoshida, Y. Takemoto, Synlett 2004, 540; b) H. Miyabe, R. Asada, Y. Takemoto, Tetrahedron 2005, 61, 385.

[23] J.-Y. Liu, Y.-J. Jang, W.-W. Lin, J.-T. Liu, C.-F. Yao, J. Org. Chem. 2003, 68, 4030.

[24] S. Bazin, L. Feray, N. Vanthuyne, M.P. Bertrand, Tetrahedron 2005, 61, 4261. 